\title{
EchoGéo
}

$53 \mid 2020$

Dénominations plurielles. Quand les noms de lieux se concurrencent

\section{Urbanisme de rattrapage, marquage territorial populaire et conflits d'odonymies dans les quartiers de Yaoundé (Capitale du Cameroun)}

Corrective urban planning, popular territorial marking and odonymic conflicts

in Yaounde (Cameroon capital)

\section{Gaston Ndock Ndock}

\section{(2) OpenEdition}

Journals

\section{Édition électronique}

URL : https://journals.openedition.org/echogeo/20168

DOI : 10.4000/echogeo.20168

ISSN : 1963-1197

Éditeur

Pôle de recherche pour l'organisation et la diffusion de l'information géographique (CNRS UMR 8586)

Référence électronique

Gaston Ndock Ndock, « Urbanisme de rattrapage, marquage territorial populaire et conflits d'odonymies dans les quartiers de Yaoundé (Capitale du Cameroun) », EchoGéo [En ligne], 53 | 2020, mis en ligne le 25 octobre 2020, consulté le 10 août 2021. URL : http://journals.openedition.org/ echogeo/20168; DOI : https://doi.org/10.4000/echogeo.20168

Ce document a été généré automatiquement le 10 août 2021.

EchoGéo est mis à disposition selon les termes de la licence Creative Commons Attribution - Pas d'Utilisation Commerciale - Pas de Modification 4.0 International (CC BY-NC-ND) 


\title{
Urbanisme de rattrapage, marquage territorial populaire et conflits d'odonymies dans les quartiers de Yaoundé (Capitale du Cameroun)
}

\author{
Corrective urban planning, popular territorial marking and odonymic conflicts \\ in Yaounde (Cameroon capital)
}

Gaston Ndock Ndock

Cette contribution est l'un des fruits du Symposium International tenu à Niamey au Niger du 5 au 10 septembre 2018 sur le thème Nommer les lieux en Afrique : enjeux sociaux, politiques et culturels. Pour un observatoire des néotoponymies urbaine, géopolitique et numérique. Je tiens à remercier très sincèrement le Professeur Frédéric Giraut de l'Université de Genève, coorganisateur de ce symposium, qui s'est particulièrement investi dès le début à l'écriture et dans la ré-écriture de certains segments de cette publication.

\section{Introduction}

1 Globalement issues de la volonté administrative coloniale, la genèse de la ville moderne africaine a posé les jalons d'une dichotomie odonymique. En effet, depuis la deuxième partie du XXème siècle, la forte dynamique d'urbanisation se heurte à l'exigence d'un encadrement urbanistique des villes. La raison se trouve, d'une part, dans la pratique coloniale, reprise par l'Etat décolonisé, d'assigner unilatéralement des noms aux villes planifiées, à leurs quartiers, voies et équipements (Bigon 2016; Bigon et Njoh 2015 ; d'Almeida-Topor, 1996 ; Giraut et Antheaume 2012 ; Goerg 2006) et, d'autre part, à une croissance démographique qui pousse à occuper des portions territoriales non aménagées, ni désignées, et encore moins délimitées par le pouvoir (Meliki, 2020). Ce double constat structure ainsi les hypothèses sur les modalités de dénomination des espaces urbains qui conduisent à un dualisme odonymique structurel. En fait, la 
production de l'espace urbain au Cameroun, à titre principal, relève aussi des « faiseurs de ville » (Paquot, 2010) informels qui y produisent une urbanisation populaire. Celle-ci est marquée par la succession Occupation/Construction pour les bidonvilles, ou Construction/Occupation/Régularisation pour les quartiers informels auto-lotis (Giraut et Rochefort, 2006). La dénomination des lieux pour des raisons pratiques de localisation, de repérage et de déplacement s'effectue ici en dehors de toute organisation. Elle relève donc aussi d'une production populaire pour usage vernaculaire (Amougou Mbarga, 2013 ; Bertrand 2001 ; Garakcheme, 2011 ; Leimdorfer et al., 2002 ; Wanjiru et Matsubara, 2016 et 2017).

2 Mais, ces quartiers populaires sont souvent progressivement intégrés au circuit urbain conventionnel, par les autorités, grâce au concours de fonds externes (Bopda, 2001) ; situation qui aboutit souvent à dénommer pour renommer ces espaces, créant ainsi une "polyphonie » (La Soudière, 2004). Lorsque ces espaces sont tirés des schèmes populaires d'organisation et arrimés aux normes d'une planification officielle, il y a, au moins dans la signalétique et la cartographie, destructuration ou effacement des repères et symboles populaires antérieurement établis par un pouvoir qui tient à rebaptiser les lieux selon les canons de l'officialité reposant sur diverses références (Antheaume, 2008 ; Bopda, 2001 ; Njoh 2010).

3 La focale sur la restructuration urbaine ${ }^{1}$ impose l'entrée par l'urbanisme de rattrapage en tant qu' « urbanisme de la transformation » qui s'opposerait à un " urbanisme de création et d'extensions périphériques » (Chaline, 1999). Il est caractérisé par la volonté des autorités gestionnaires de la ville de reprendre la main sur des segments territoriaux qui accentuent le visage d'une ville en crise (Stadnicki, 2009).

Dès lors, c'est à l'interface de cette double dynamique populaire de production urbaine et d'urbanisme de rattrapage des pouvoirs publics que cette réflexion, envisageant une analyse processuelle, se structure pour répondre à la question de savoir quels sont les enjeux qui président à la formation d'une toponymie polyphonique de la ville de Yaoundé. L'hypothèse formulée est que la production urbaine populaire relève d'une toponymie vernaculaire pratique, là où les couches officielles ultérieures relèvent de l'imposition de référentiels fondateurs pour les pouvoirs publics ainsi que d'une volonté d'effacement des marques vernaculaires (Giraut et al., 2008). On émet également l'hypothèse que ces dernières sont cependant maintenues dans les pratiques par attachement au registre vernaculaire et populaire et défiance vis à vis des référentiels officiels abstraits.

5 L'effort réflexif vise ainsi à démontrer, à partir du cas de Yaoundé, ville capitale politique du Cameroun, comment du choc des dynamiques urbaines du bas et de l'officialité, jaillissent des systèmes toponymiques qui s'affrontent du fait des références, des représentations et d'enjeux de pouvoir contradictoires (Guillorel, 1999), d'où la polyphonie. Le travail articule deux autres faits majeurs. D'abord, le pouvoir s'empare du champ toponymique pour opérer un marquage politique, symbolique, idéologique ou mémoriel du territoire en fonction de ses référents et intérêts de légitimation politique. Ensuite, pour les populations, une contestation des toponymies officielles vécues comme une perte d'identité socio-spatiale au profit de référents étrangers. 


\section{Considérations méthodologiques}

6 Yaoundé est le terrain d'une enquête qui a mobilisé observation, entretiens et l'analyse documentaire.

\section{Des données issues des techniques vivantes de collecte}

7 Tout d'abord, des observations directes répétées ont été menées dans deux types d'espaces à Yaoundé. D'une part, des quartiers nouvellement créés du fait des logiques opportunistes des "gens d'en bas" (Ela, 1998). La circonscription administrative de Yaoundé IV a pour cela été choisie. D'autre part, des quartiers d'habitations populaires qui font l'objet d'une restructuration par le pouvoir central à Yaoundé II. Il s'agissait de cerner et de comprendre, en fonction du toponyme, les rapports entre odonyme et éléments du milieu, les personnes ou des faits fondateurs des lieux. Ensuite, mobilisé pour approcher des acteurs-clés, l'interview a été menée avec seize personnes, à raison de sept sur le premier site et de neuf sur le second. Une diversification genrée des enquêtés a été effectuée, notamment avec la présence de cinq femmes contre onze hommes. La faiblesse relative des femmes tient à leur faiblesse statistique comme propriétaire de maison ou chef de ménage. Les catégories de chef de secteur, chef de quartier, propriétaire de maison, patriarche et autorités municipales identifient les personnes-ressources. Exception faite des autorités, les enquêtés appartiennent à des classes sociales indigentes et habitent des quartiers populaires. Des objectifs spécifiques étaient poursuivis à travers l'interview. D'abord, glaner les informations sur les dénominations des lieux, dans l'optique de saisir la dynamique de délimitation et les référentiels qui engendrent ces noms. Ensuite, il s'est agi de circonscrire à partir des représentations sociales, les enjeux dont sont porteurs les odonymes d'inspiration populaire ou officielle. Les données documentaires ont complémenté ces informations.

\section{Mobiliser des sources de données documentaires et géométriques}

8 Les cartes administratives, certaines données sur la dénomination des unités administratives (toponymes), de reliefs (oronymes), des cours d'eaux (hydronymes), des ethnies (ethnonymes) ont été acquises à l'Institut National de la Cartographie (INC) du Cameroun et au Ministère de l'administration territoriale (MINAT). De même, la première structure a-t-elle permis d'obtenir des données géométriques, notamment des informations issues du Système d'Information Géographique national, de la mosaïque administrative du Cameroun et des cartes topographiques. Le réseau hydrographique a été étudié afin de mettre à jour l'homophonie entre cours d'eau et secteurs de résidence. Le même travail a été effectué pour la carte topographique. Dans la mesure où la problématique de l'odonymie des quartiers est liée aux récits, aux projections et aux rapports à l'environnement naturel, le paradigme des représentations sociales s'est imposé comme cadre d'interprétation des données recueillies, par la mobilisation des constructions symbolique et mentale que les acteurs développent sur un objet (Fischer, 1983 ; Jodelet, 1989). 


\section{La crise urbaine comme matrice du conflit des référents identitaires a Yaounde}

9 Yaoundé entretient une croissance soutenue avec une taille moyenne des ménages estimée à 5,5 personnes (PDU, 2008 : 19). Du côté des financements, les gestionnaires de la ville font un constat: "L'État [...] ne dispose pas de suffisamment de fonds pour financer la modernisation de Yaoundé ${ }^{2}$, vu que les principales sources de financement étaient basées sur des programmes d'aide aux pays endettés (le C2D, par exemple) ${ }^{3}$, aujourd'hui épuisés. Le tissu urbain se construit alors sur une logique anarchique de l'occupation de l'espace, expressive d'une absence de planification urbaine ${ }^{4}$.

\section{Une politique d'urbanisation et de planification urbaine inopérante}

10 Le phénomène migratoire qui fait de la capitale un lieu de convergence de populations et de naissances nombreuses, explique partiellement l'ampleur des installations anarchiques. Yaoundé se définit comme "une ville trajective» (Berque 2000). C'est donc un lieu de convergence des populations, mais aussi de leur redistribution vers les périphéries. Déversoir démographique, la ville est alors l'objet de sollicitations et d'initiatives d'investissements immobiliers. Du fait du caractère continu de ce processus, les autorités municipales, débordées, ne peuvent au préalable organiser, viabiliser et dénommer ces espaces qui sont ainsi urbanisés de manière spontanée. Ce fait doit ici être corrélé à un dyptique causal majeur issu de la variable migratoire : l'expansion spatiale et démographique et, le caractère exsangue des budgets d'investissement urbain. Sur les plans démographique et spatial, la convergence des masses à Yaoundé entretient une croissance soutenue (Bopda, 2003). Les projections du PDU prévoyaient 3400000 habitants en 2020, le corollaire est donc une sortie des limites du tissu urbain initial, estimé en 2002 à 9924 hectares pour une superficie totale de 30 954,18 hectares. Aussi, les structures d'aménagement urbain se retrouvent-elles face à un double problème : une dynamique métropolitaine qui s'effectue au-delà des anciennes limites du périmètre urbain et les pratiques populaires de colonisation de nouvelles surfaces considérées comme non constructibles - cette catégorie totalise plus de 9 493,02 hectares (PDU 2008:54) - et des terres dites périurbaines. Dès lors, une fois rappelé que les gestionnaires de la ville martèlent le fait que « L'État [...] ne dispose plus de suffisamment de fonds pour financer la modernisation de Yaoundé $»^{5}$, une planification urbaine anticipatrice reste impossible.

11 De ces éléments, on peut formuler le double constat d'une faillite et du manque d'application des documents de planification urbaine : SDAU, PDU, POS, PSU, PDL. De même, la prise d'assaut populaire de secteurs et de friches urbaines met en évidence l'absence de politique urbaine et d'un pilotage stratégique de l'aménagement urbain. Subséquemment, l'arrivée massive et permanente de populations, met à mal le pouvoir central et les municipalités dans leur prérogative d'ordonnancement et de planification de la dynamique d'urbanisation (illustration 1). Dès lors, le caractère inopérant de la planification urbaine a pour façade tangible la production d'ensembles résidentiels où il n'existe ni servitude, ni réseau de distribution d'énergie électrique, ni service d'adduction d'eau potable, encore moins d'odonymes consacrant une dénomination officielle des lieux. Ces constats soulignent ainsi les logiques opportunistes des "exclus 
de la civilisation urbaine" qui tentent d'accéder à la propriété immobilière et au mode de vie urbain (Meliki, 2020).

Illustration 1 - Croissance spatiale et démographique de Yaoundé de 1956 à 2005 : une analyse multiscalaire et situationnelle

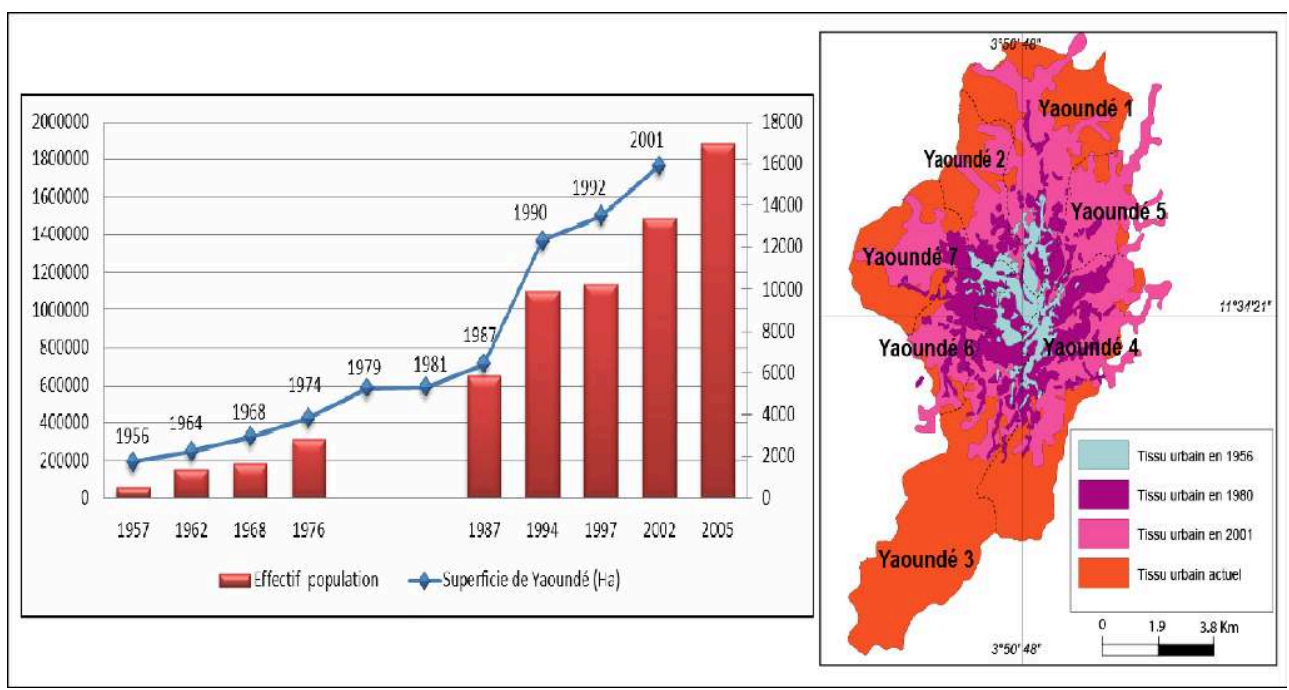

Source : Conception Olivier Maurice Zoning Moffo. Réalisation : Gaston Ndock Ndock, 2012.

\section{Les logiques opportunistes d'installation des populations dans l'espace urbain}

12 Les populations venues de l'arrière-pays et un taux de croissance démographique de $2,37 \%$, génèrent un phénomène de saturation du noyau urbain de Yaoundé, une rareté du sol pour les particuliers et la puissance publique, d'où une cherté du foncier valorisé et aménagé. Il s'ensuit une logique censitaire dans le marché foncier conventionnel et une flambée du coût de la vie pour les indigents. Ces réalités acculent les citadins pauvres à des logiques opportunistes.

13 Cela étant, le « théorème du volcan » qui marque l'urbanisation de la capitale, repousse en périphérie la part la plus indigente des populations en quête d'opportunités foncières accessibles (Lacour, 1999). Les nouveaux sites ainsi urbanisés tiennent de la disponibilité en terres de faible valeur et de la multiplicité des mécanismes d'acquisition conciliants pour les classes précarisées. Il s'agit des couronnes périurbaines, terres situées dans l'entre-deux, précisément au point de sortie des campagnes ou des villes satellites et à l'amorce du tissu urbanisé (Certu, 2007; Yemmafouo, 2013). Ce sont des « espaces compris entre l'agglomération morphologique d'un côté, et la campagne rurale et ses localités de l'autre » (Bryant et Marois, 1998) ; les interstices urbains, désignant un résidu spatial, un vide urbain ou une zone d'abandon constitutif d'une transition entre deux blocs de construction ou deux espaces planifiés (Rivière, 2012); les espaces classés non constructibles qui intègrent de vastes terres hydromorphes - marécages, bas-fonds - et des zones accidentées ainsi que les terres semi-rurales sans valeur ponctuelle pour les autorités.

Il faut en effet relever que ces espaces sollicités par les indigents se situent en marge des fronts d'urbanisation envisagés par la planification urbaine. Ces secteurs ont initialement un caractère semi-rural de par le désintéressement des politiques urbaines 
à leur endroit. Au stade urbain embryonnaire, ce facteur qui justifie l'absence de réglementation et la facilité d'accès à une parcelle de terres, devient l'adjuvant d'un déferlement des exclus. En fait, les valeurs sur ce marché foncier peu formalisé, relevant $\mathrm{du}$ droit coutumier, sont de loin inférieures aux prix des canaux conventionnels d'accès au foncier valorisé.

15 Ainsi, l'opportunisme dans l'acquisition d'une propriété foncière est à coupler à la faiblesse du niveau de vie, comme moteur qui commande l'irruption au sein de ces espaces semi-rustiques aux prix de denrées alimentaires, de biens et services artisanaux abordables. Ces facteurs exercent une forte attraction sur les migrants et exclus de la métropole, accélérant ainsi la construction de nouveaux secteurs résidentiels sans PDU et POS, encore moins une politique de desserte et de fourniture en services sociaux de base. Face à l'incapacité des pouvoirs publics à fournir des services et équipements nécessaires, les zones ainsi investies par les «laissés-pourcompte de la civilisation urbaine » se retrouvent au centre d'initiatives et de stratégies populaires alternatives.

\section{Production urbaine par le bas et système d'odonymie populaire}

Les formations spatiales hors de l'emprise des pouvoirs publics obéissent à des systèmes de localisation et de dénomination qui décrivent et racontent ces lieux. Dans cette production populaire des odonymes urbains, les déplacements par les moyens de transports alternatifs ou précaires (moto-taxis) participent dans la diffusion des odonymes. Car, prenant pour repère des particularités des lieux. Les champs de référencement qui donnent sens à ces odonymes relèvent de narratifs qui restituent les dynamiques socio-spatiales majeures des quartiers considérés. De plus ils s'effectuent dans ce que Louis Martin Onguene Essono (2018) appelle «un volcan linguistique actif ».

\section{Une toponymie expressive des particularismes du milieu physique}

17 L'entreprise populaire de dénominations secrète des toponymes qui disent la vie sociale ou décrivent les traits marquants de l'environnement. L'odonyme est ainsi un discours sur le milieu. Il renferme des informations dont l'intelligibilité n'est accessible pour autant que l'on puisse comprendre le champ référentiel qui l'irrigue, ce qui implique une capacité de décryptage des codes liés aux systèmes langagiers en vigueur. Le toponyme a une charge sémiotique qui renvoie à une langue, un référent et un contexte spécifique (Boutaud, 1998).

En ce sens, les mécanismes de référenciation en jeu puisent une part de leur substance dans les repères géographiques de l'espace considéré. Ici, la relation entre désignant et désigné, c'est-à-dire l'odonyme et sa référence matérielle ou mentalement construite met en lumière une structure topographique particulière ou le caractère unique d'un élément du milieu. Dès lors, le langage populaire, à travers les idiomes locaux et parfois l'argot, dit la géographie locale. Cette construction mentale et imagée d'une réalité via la toponymie est liée aux mentalités et pratiques spatiales et sociales (Jodelet, 1989; Mucchielli, 1985). 
A cet effet, rappelons que Yaoundé est lui-même appelé Ongola-Ewondo, c'est-à-dire la clôture des Ewondos. Ce substantif, issu de la langue vernaculaire, désigne un habitat constitué de "paillotes" de forme rectangulaire protégées par des enclos faits d'arbustes: descriptif essentiel du pays des Ewondo (Leques, 1955, p. 33). Une telle grammaire nominative qui puise dans des éléments anthropologiques - groupe culturel en présence - et le visuel architectural externe de l'habitat dominant - type de case et leur aspect - consacre la logique sémio-contextuelle qui sert partout d'ancrage au système odonymique vernaculaire.

À Yaoundé, nombre de dénominations populaires sont puisées dans le répertoire de noms existants, relatifs à l'environnement et au relief. C'est ainsi que l'investissement d'un espace interstitiel, de nature non constructible pour le pouvoir, entre une zone commerciale (Bata), et un espace résidentiel pour classes modestes (nkol-Eton), s'est vu dénommer : nlongkak-Elobi, par les indigents, désignant sa structure marécageuse ; elobi étant ici un mot de l'idiome Ewondo qui désigne les bas-fonds marécageux. Dans le même ordre, on retrouve des éléments topographiques dans ces dénominations. C'est ainsi que des odonymes dépeignent la structure collinaire ou montagneuse saillante de leurs milieux. Les populations vont baptiser leurs espaces à partir du mot ewondo «nkól », signifiant colline, lequel remplit les rôles de préfixe et de radical (Essono, 2016, p. 352). Des noms de quartiers populaires tels que mbankolo et nkolbikok souscrivent à ce registre. Cette relation entre particularité environnementale et toponymie irrigue les phytonymes et zoonymes. C'est respectivement les cas du quartier populaire de Oyomabang (vieil Iroko) et Djoungolo (caméléon), espèce faunique prégnante.

Ainsi, il y a donc production d'une richesse odonymique ancrée dans les éléments de l'environnement naturel et historique, mais aussi à la vie sociale contemporaine. L'illustration 2 présente ainsi des quartiers, des rues et des habitations adressés suivant une nomenclature informelle ou illégale dans la ville de Yaoundé.

Illustration 2 - Des quartiers, des rues et des habitations adressés suivant une nomenclature informelle ou illégale
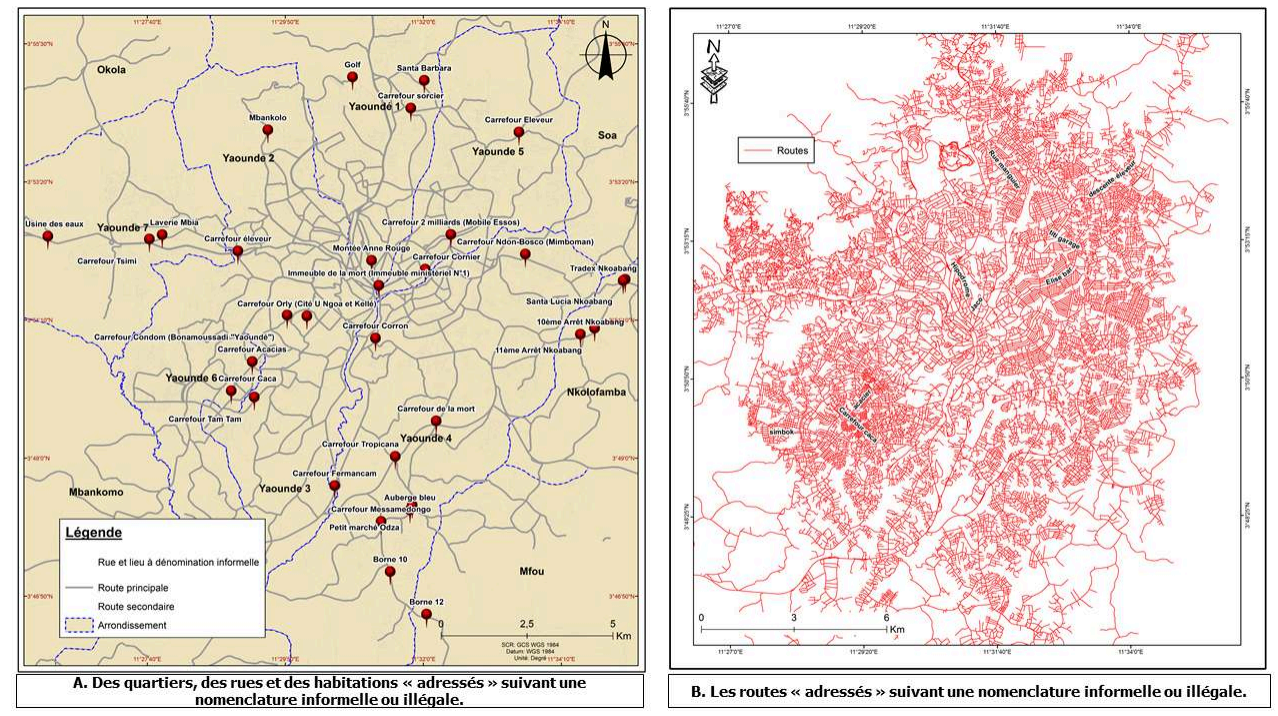

Sources : Communauté urbaine de Yaoundé, 2014 et observations de terrains, 2017. 


\section{L'histoire sociale des lieux au cœur des dénominations}

après les analyses effectuées à Yaoundé, métropole politique et Douala, métropole économique, la toponymie est un narratif dynamique qui dit la longue durée et la quotidienneté (Amougou Mbarga, 2013). Dans une perspective historique, une majorité des noms de quartiers de la ville de Yaoundé raconte les séquences de la mise en place des populations. Ils rendent compte de la mise en valeur d'un espace par une tribu ou une figure ancestrale d'un clan, d'où l'existence de toponymes incluant les termes mvog ou elig dans la dénomination des quartiers (Essono, 2016 : 370). Chaque clan est désigné par mvog - qui renvoie à « clan de » - ou par elig, c'est-à-dire la « descendance de ». On verra donc des quartiers comme : Mvog-Ada, Mvog-Mbi, Mvog-Betsi, Elig-Edzoa, Elig-Essono, Elig-Belibi, entre autres. Ces toponymes, de nature patronymique, rendent compte de l'histoire socio-culturelle des quartiers désignés à travers la filiation et la culture ethnique contenues dans le désignant.

Ce constat historique met en contexte un même processus de dénomination actuelle des quartiers spontanés. Les noms de quartiers contemporains d'émanation populaire prennent en charge des faits et des personnes qui font références pour l'identité de nouveaux territoires urbains. Les toponymes sont ici dépositaires de la mémoire collective récente des lieux. Des dénominations de quartiers telles que «carrefour Happi » dans l'arrondissement de Yaoundé IV, "Carrefour sorcier " à Yaoundé $\mathrm{I}^{\mathrm{er}}$, "Carrefour Caca» de l'arrondissement de Yaoundé VI, "Carrefour Condom» ou "Vallée de la mort » respectivement situés à Yaoundé III et I ${ }^{\text {er }}$ dont les exégèses sont faites à la section suivante, procèdent de cet esprit. Le premier renvoie à un entrepreneur qui a marqué la vie du site. Capitaine d'entreprises, ses affaires ont rythmé l'économie du quartier. Le second doit son nom à des évènements paranormaux récurrents : il est un lieu culte de départ et d'arrivée de vols occultes ayant cependant des manifestations tangibles comme le rapporte en détail la prochaine section. Le troisième renvoie à un problème d'hygiène et de salubrité: des excréments qui proviennent en grande quantité des fosses septiques, se déversent dans la rue. Depuis une dizaine d'années, les habitants des quartiers (Biyem-Assi, Acacias, Jouvence) de l'arrondissement de Yaoundé VI pataugent régulièrement dans ces eaux usées. Une quinzaine de fosses septiques connectées aux logements avoisinants ont été construites au milieu de la route principale. Malgré leur proximité avec des logements administratifs et sociaux, et même un marché, elles ne sont pas entretenues et laissent échapper sur la chaussée leur contenu, une fois les cuves pleines. Le quatrième mettrait en exergue une certaine débauche sexuelle dans un quartier typiquement estudiantin, alors que le dernier toponyme renseigne sur une tranche sanglante de l'histoire politique du Cameroun. Il pointe le lieu où furent construites des geôles, haut lieu de tortures et d'exécution sommaires des dissidents.

Ces toponymes sont une fenêtre sur l'histoire et les usages des espaces (illustration 2). Ils dépeignent des faits et des figures autour desquels se sont construits les territoires en question (Guillorel, 2012). C'est d'ailleurs pourquoi, il existe une variation temporelle et conjoncturelle des toponymes sur les sites observés comme suggéré à la prochaine section. Partout en zones urbaines camerounaises, la toponymie d'émanation populaire est un discours sur le temps social, politique et culturel de la population. Le «Carrefour maquisard » à Bafoussam tient ainsi son nom de l'événement d'exécution sommaire des derniers résistants nationalistes à l'Ouest Cameroun 
(Mbembe, 1989). Le "Carrefour Trois voleurs " et le carrefour «J'ai raté ma vie » à Douala, le "Carrefour Tamzou » à Ebolowa rappellent des déviances sociales observées dans ces villes (Pangop Kameni, 2006). Les toponymes soulignent donc la brutalité du pouvoir central et la supposée dépravation des mœurs, moyen de survie alternatif qui accuse, par-là, l'incapacité du pouvoir à produire les conditions d'emplois rémunérés pour la population. C'est pourquoi, le pouvoir, brocardé, contesté et dérangé par ces toponymes, essaie d'attribuer de nouvelles dénominations à la faveur des opérations de restructuration urbaine.

\section{Représentations sociales distinctives et polyphonie toponymique} postériori des installations spontanées et auto-organisées de populations (Nzuzi, 1989), autorise une réorganisation du territoire. Cette "modernisation " s'exprime souvent par des déguerpissements ou de simples restructurations sur fond d'assainissement basique (Chenal et Diagana, 2016). Elle se prolonge aussi dans le domaine toponymique souvent dans l'ignorance des repères populaires qui s'opposent alors à la strate officielle.

\section{Enjeu d'adressage et affrontement de dénominations à partir de registres symboliques différenciés}

La rationalité statistique et numérique est au centre de l'urbanisme de rattrapage. Elle s'inscrit en droite ligne des recommandations globales d'adressage par les organisations internationales en matière de production de villes intelligentes (Njoh, 2010). Adossée à la gouvernance par les chiffres, cette logique met en jeu, dans les dix chefs-lieux de régions, la généralisation d'une logique du découpage et du décompte pour une meilleure organisation spatiale. Ainsi, malgré l'absence relative de sa mise en œuvre, les dix Communautés Urbaines, devenues Mairies de ville à la faveur de la loi n²019/024 du 24 décembre 2019 portant code général des collectivités territoriales décentralisées, essaient de se doter chacune d'un PDU. À Yaoundé ou à Douala, tel que mené par les Mairies de ville, l'urbanisme de rattrapage pose les jalons de cette gouvernance urbaine qui produit trois résultats principaux. D'abord, une certaine valorisation $\mathrm{du}$ foncier liée aux travaux de viabilisation répondant aux standards, même primaires, des prescriptions urbaines en la matière. À Yaoundé, à titre illustratif, le bloc Mbankolo-Chefferie, naguère malfamé, passe au rang de nouveau quartier convoité à cause des travaux menés. Après la construction de larges routes bitumées à double-sens, de ponts et l'instauration de l'éclairage public, des conflits sont observés entre le collectif de défense des terres du quartier et la société d'investissement immobilière, Clemevesf. Celle-ci désire racheter de force les terrains pour rallonger la zone résidentielle dédiée principalement aux diplomates et à la bourgeoisie d'affaire. Autre effet de ces politiques : la réorganisation territoriale des espaces aménagés. Le quartier Mbankolo-Chefferie est ainsi intégré sur la carte de la ville et pratiquement annexé au bloc des classes aisées du quartier Golf qui jouxte le vaste domaine de la Présidence de la République du Cameroun. Enfin, la fourniture de commodités et la réalisation d'une trame viaire normalisée permet une systématisation du repérage des

EchoGéo, 53 | 2020 
voies et des parcelles selon une logique numérique. Ainsi, les opérations de restructuration urbaine sont souvent couplées à l'attribution de nouveaux odonymes, le pouvoir tenant à rebaptiser selon les canons de l'officialité les quartiers restructurés (illustration 3). Mue par des intérêts et une idéologie distincte des logiques populaires, l'autorité s'empare parfois du champ odonymique pour opérer un marquage symbolique, idéologique ou mémoriel du territoire, voire pour banaliser ou déshistoriciser les lieux dédiés à des figures qui « dérangent ", ou encore pour légitimer ou justifier une création spatiale (Veschambre, 2004 ; Bertrand 2004). Cette posture contrarie la volonté populaire de maintien des toponymes vernaculaires liés à la construction symbolique et mentale de l'espace.

Si le droit de nommer est le versant linguistique du droit de s'approprier (Calvet, 2002), attribuer des noms devient, pour le pouvoir, un acte politique qui affirme son impérium (Giraut et al., 2008), légitime son emprise et sa politique du city marketing (Borja, 1996) ou du City Development Strategy (CDS), tandis que pour la population, il s'agit de pérenniser une mémoire environnementale, un socle culturel ou humain qui restitue une tranche d'histoire significative. Les renominations officielles sont donc créatrices de dissensions. Il peut s'en suivre des incompréhensions qui mènent à récuser les appellations officielles et à rebaptiser les segments désignés par l'autorité tout en engageant des actes de vandalisme envers la nouvelle signalétique officielle (Guyot et Seethal, 2007).

Dans la ville de Yaoundé, odonymes officiels et populaires s'affrontent. Le quartier "Manguier" au lieu-dit Grand Canyon bar, abrite un carrefour célèbre. Sporadiquement, on y découvre au petit matin des personnages dénudés perchés sur les pilonnes électriques, placés sur les dômes d'immeubles ou simplement encastrés dans les fentes d'un mur de maison. Fouettés ou menacés, ces personnages font la confession de vols occultes nocturnes menant à un « atterrissage d'urgence » dû à la lumière du jour : tel est l'origine de "Carrefour Sorcier». Toutefois, l'État, dans sa raison cartésienne, a tenté de rebaptiser le lieu en "Carrefour Ody ». De même, les populations ont-elles débaptisé le «Carrefour Mobil Essos» au courant de l'année 2018 pour imposer " Carrefour 02 milliards ». Cet odonyme vient d'un acte de "générosité » d'une dame installée de bonne heure dans un supermarché jouxtant ce carrefour, se proposant de régler les factures de tous les clients. Le pouvoir réfutera cette appellation en maintenant, dans la publicisation de ses évènements et autres activités à cet endroit, l'appellation originelle. Il faut aussi rapporter le cas des quartiers Biyem-Assi, Jouvence, Acacia dans l'arrondissement de Yaoundé VI dont les rigoles des carrefours sont parfois envahies par les déjections humaines venant des fosses septiques et rendant irrespirable l'air du lieu. "Carrefour Caca» est alors l'odonyme trouvé par les populations pour ces lieux. Ce nom est repoussé par le pouvoir qui oppose toujours le toponyme de "Montée Jouvence». Les populations maintiennent alors «Carrefour Caca » pour rappeler l'omniprésence des selles et l'incapacité des municipalités à assainir ce quartier. Cette dynamique est aussi observée à Douala. Dans le quartier "Village ", haut lieu de réjouissance populaire, la logique festive, l'insouciance, la débauche sexuelle, l'ivresse et la criminalité, justifient le nom Carrefour «J'ai raté ma vie ». Le pouvoir a plusieurs fois posé des plaques pour récuser cette appellation en faveur de celle de "Carrefour Nelson Mandela ", mais les habitants et usagers des lieux l'ont récusé. C'est dans la même foulée que l'on note chaque année, à Douala, des «tentatives répétées de démolition de la statue du général Leclerc par Mboua Massock, 
personnage bien connu de la scène publique et politique camerounaise " (Ndjock Nyobe, 2014).

Illustration 3 - Quartiers désignés suivant une nomenclature légale ou officielle
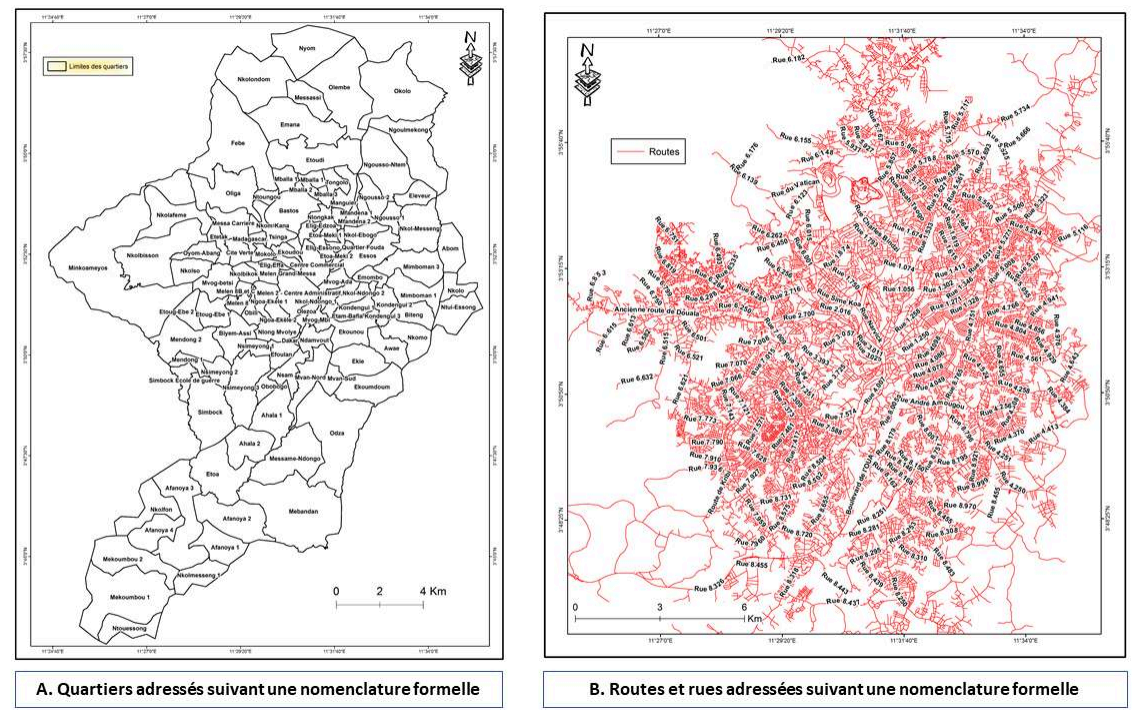

Sources : Communauté urbaine de Yaoundé, 2014, observations de terrains, 2017.

les sanctions pécuniaires infligées - que la diaspora camerounaise s'empresse de payer - et les peines de prison ferme, déboulonne la statue du général Leclerc et essaie de la remplacer par des noms et bustes des nationalistes comme Um Nyobè, père de l'indépendance, mais aussi des acteurs politiques phares tel que John Ngu Foncha, principal acteur du processus de réunification des deux Cameroun. En effet, la statue du général Leclerc, située à la place de l'Indépendance au lieu-dit "poste de Bonanjo ", rappelle un passé colonial douloureux et des transactions politiques au profit du bloc dirigeant actuel. Elle fait ainsi ombrage aux héros nationaux et régionaux (Ndjock Nyobe, 2014). Déboulonner cette statue, est ici une forme d'expression politique qui va de pair avec une revendication de toponymes et symboles populaires.

Avec la restructuration des quartiers à Yaoundé, des toponymes et figures étrangères font irruption. Tel est le cas aussi du «Carrefour Monseigneur François Xavier Vogt ", flanqué d'un buste le représentant, ou de "l'Avenue Jean-Paul II ». Le pouvoir s'attèle, par cette technique, à faire disparaitre les odonymes considérés comme péjoratifs ou populaires. Ces pratiques et les réponses populaires d'usage et d'affirmation des appellations vernaculaires débouchent sur une polyphonie dont l'enjeu est le pouvoir.

\section{La nomination des lieux au-delà des enjeux identitaires et mémoriels, une question de pouvoir}

Baptiser un lieu ou en changer le toponyme est un acte de pouvoir (HoussayHolzschuch, 2008). La topographie urbaine et les toponymes qui en désignent ses éléments et parties, entrent dans le champ de la projection du pouvoir (Tchumtchoua et Ndjock Nyobe, 2013). Dans un contexte où le pouvoir, possibilité d'agir sur un individu, s'inscrit dans l'ordre de la relation entre individus (Bocher et al., 2010, p. 237), 
l'acte de dénomination devient prescriptif d'une volonté, d'une histoire et d'une manière de concevoir l'environnement et ses individus. Il s'agit d'un rapport de force. Le nom est ainsi un attribut du territoire; il le désigne, le situe, le qualifie, symbolise sa substance (Austin, 1963, Claval 2003) et (re)écrit son histoire dans un sens favorable à l'acteur qui l'initie. Ainsi, des enjeux politiques se dégagent une fois rappelée l'histoire du bloc dirigeant actuel qui cherche à imposer son autorité, à façonner l'histoire politique à son avantage et implémenter sa politique urbaine. De ce point de vue, l'indépendance a débouché sur un conflit entre ex-colonisés à propos du passé. Le pouvoir, soucieux de se fabriquer une légitimité que lui ôtaient de facto les évènements liés à la décolonisation, essaie de reconstruire une nation déblayée de tout conflit symbolique. Et, pour cela, utilise de manière sélective des figures et des évènements conciliants pour dénommer les quartiers et places, imposant alors une amnésie collective officielle sur les figures nationalistes populaires qui auraient pu hériter du pouvoir (Mbembe, 1989).

Face à ce dispositif toponymique qui rend compte de l'emprise du pouvoir, le versant contestataire populaire, considéré dans la section précédente, exprime le rejet d'odonymes unilatéralement assignés par un pouvoir post-autoritaire. Ces faits justifient aussi la polyphonie observée dans les villes. Ainsi, pour une part de la société civile, toute toponymie en rapport avec l'indépendance ne serait acceptée qu'en restituant son contenu symbolique et historique effectif. C'est pourquoi, elle s'attaque souvent frontalement au diktat mémoriel et historique véhiculé par la toponymie d'Etat. Par ailleurs, les populations revendiquent des odonymes qui disent une réalité socio-culturelle, identitaire ou politique admise du lieu. Ainsi donc, le «Carrefour sorcier ", malgré un acte officiel faisant de lui le "Carrefour Ody ", conservera son appellation originelle pour la population, tout comme la construction du monument de «La nouvelle Liberté » à Douala ne parvient pas à changer nominalement le "RondPoint Deïdo ». Pour certaines populations, le maintien des toponymes qu'elles secrètent devient l'expression d'une double réalité : un acte de citoyenneté et un mouvement contestataire. L'élan de citoyenneté traduit le refus de perte d'une identité sociospatiale devant un pouvoir enclin à l'adoption de référents étrangers aux représentations vernaculaires. Ainsi, les analyses développées ci-dessus suggèrent que la logique oppositionnelle mettant aux prises autorité et populations au sujet du contrôle de la toponymie ne peut être dissociée de la question du pouvoir. L'illustration 4 ci-dessous est une synthèse des nomenclatures officielles et nonofficielles des quartiers de Yaoundé. 
Illustration 4 - Synthèse des quartiers de Yaoundé désignés suivant une nomenclature officielle et non officielle

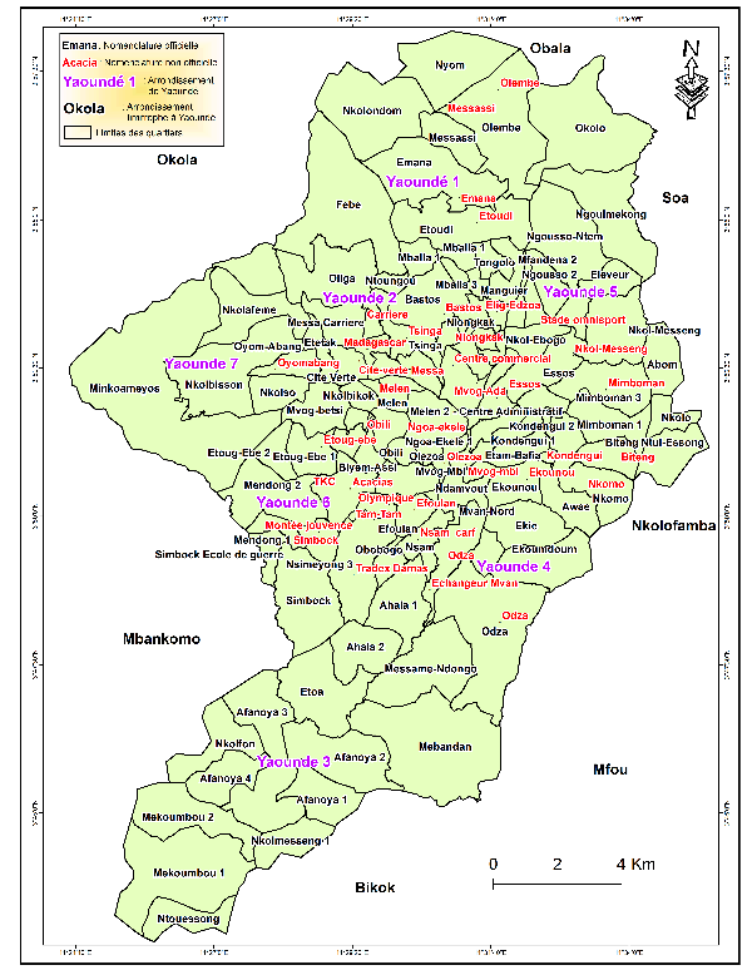

Sources : Communauté urbaine de Yaoundé, 2014, observations de terrains, 2017.

\section{Conclusion}

Ce travail questionnait les facteurs et enjeux qui rendent compte, dans le contexte camerounais, à partir du cas de la ville capitale de Yaoundé, d'une polyphonie odonymique, opposant toponymies urbaines vernaculaires et officielles. À partir des données de terrain, il est confirmé que les deux moteurs de la production toponymique urbaine sont d'une part l'urbanisation spontanée informelle et le maintien de sa logique et d'autre part la volonté de restructuration urbaine de l'autorité à postériori désignée par le concept d'urbanisme de rattrapage. De ce diptyque causal, il apparaît que les deux logiques de production urbaine sont génératrices d'une toponymie duale et contradictoire. En tant que messages qui témoigne d'éléments mémoriels et fonctionnels, les odonymes recouvrent dès lors des enjeux de pouvoir qui justifient la volonté des acteurs populaires et publics à maintenir, chacun, leur registre toponymique, vernaculaire et officiel. Cette absence de consensus autour du désigné et du signifié, ainsi que la dichotomie des référents qui légitiment de part et d'autre un ordre et une vision du monde, provoquent un affrontement qui installe une polyphonie toponymique rendant difficile l'orientation et l'adressage dans l'espace urbain métropolitain. Cela étant, un toponyme est à envisager comme un enjeu en soi, dans la mesure où son choix met en présence différents acteurs, projets, cultures, identités et représentations souvent empreintes du sceau de la conflictualité. 


\section{BIBLIOGRAPHIE}

AbdouMaliq S., 2006. Pirate towns: reworking social and symbolic infrastructures in Johannesburg and Douala. Urban Studies, vol. 43, $\mathrm{n}^{\circ}$ 2, p. 357-370.

Amougou Mbarga A. B., 2013. À travers les dénominations des rues et des quartiers de la ville de Douala : La quotidienneté comme univers de sens. Anthropologie et Sociétés, vol. 37, nº 1, p. 195-212.

Antheaume B, 2008. Les changements toponymiques sud-africains tombent parfois à côté de la plaque... L'Espace géographique [En ligne], vol. 37, n 2, p. 151-152. DOI: https://doi.org/10.3917/eg. 372.0151

Austin J.L, 1963. How to do Things with Words. Cambridge, Harvard University Press, 1963.

Benjelloun S. C., 2006. La place des langues dans l'analyse du fonctionnement spatial : la mise à jour d'interactions systémiques. Les Cahiers d'Outre-Mer [En ligne], n² 234 URL: http:// journals.openedition.org/com/409 - DOI: https://doi.org/10.4000/com.409

Bertrand M., 2001. Dynamiques urbaines, composition toponymique : le cas de Bamako (Mali). In Rivière d'Arc H. (ed.), Nommer les nouveaux territoires urbains [Nouvelle édition en ligne]. Paris, Éditions de la Maison des sciences de l'homme, URL: http://books.openedition.org/editionsmsh/ 1401 - DOI: https://doi.org/10.4000/books.editionsmsh.1401

Bigon L (ed.), 2016. Place Names in Africa. Colonial Urban Legacies, Entangled Histories. Cham, Springer.

Bigon, L., Njoh A. J., 2015. The Toponymic Inscription Problematic in Urban Sub-Saharan Africa: From Colonial to Postcolonial Times. Journal of Asian and African Studies, vol. 50, n 1, p. 25-40.

Bocher H. et al., 2010. Réseaux et pouvoir. Logiques de l'informel. Hypothèses, n 14, p. 235-246. URL: https://www.cairn.info/revue-hypotheses-2011-1-page-235.htm

Bopda A., 2001. De l'usage de fonds mythiques dans les remaniements territoriaux en Afrique et au Cameroun. Cahiers de géographie du Québec, vol. 45, n 126, p. 451-478.

Bopda A., 2003. Yaoundé et le défi camerounais de l'intégration. À quoi sert une capitale d'Afrique Tropicale? Paris, CNRS Editions, $421 \mathrm{p}$.

Borja J., 1996. Ville, démocratie et gouvernabilité : le cas de Barcelone. Revue internationale des sciences sociales, $n^{\circ} 147$, p. 1-149.

Calvet L-J., 1974. Linguistique et colonialisme. Petit traité de glossophagie. Paris, Payot, 236 p.

Charaudeau P., 1983. Langage et discours. Paris, Hachette, 176 p.

Diagana I., Chenal J., Hasler S., 2016. Villes africaines : restructuration des quartiers précaires. EPFL Press, eBook [PDF].

Claude C., 1999. La régénération urbaine. PUF, 127 p.

Claval P., 2003. Géographie culturelle. Une nouvelle approche des sociétés et des milieux. Paris, Armand Colin.

D'Almeida Topor H., 1996. Le modèle toponymique colonial dans les capitales de l'Ouest africain francophone. In Coquery-Vidrovitch C., Goerg O. (dir.), La Ville européenne outre mers : un modèle conquérant? Paris, L'Harmattan, p. 235-243. 
De La Soudière M., 2004. Lieux dits : nommer, dé-nommer, re-nommer. Ethnologie française, vol. $34, n^{\circ} 1$, p. 67-78.

Eckert A., 1999. Mémoires anticolonialistes au Cameroun. La recherche vaine de "héros nationaux. In Chrétien, J.-P. Triaud J.-L. (ed.), Histoire d'Afrique. Les enjeux de mémoire. Paris, Karthala, p. 473-487.

Essono J.-M., 2016. Yaoundé. Une ville, une histoire, 1888-2014, Encyclopédie des mémoires d'Ongola Ewondo, la Ville aux « Milles Collines». Yaoundé, Editions Asuzoa, 675 p.

Essono L.M.O., 2018. La ville de Yaoundé : un volcan linguistique actif. Sens-Dessous, vol. $21, \mathrm{n}^{\circ} 1$, p. 91-103.

Fischer G.-N., 1987. Les concepts fondamentaux de la psychologie sociale, Psycho Sup, [5 édition revue et actualisée]. Paris, Dunod, $321 \mathrm{p}$.

Franqueville A., 1984. Yaoundé. Construire une capitale. Paris, Orstom.

Garakcheme G., 2011. Références violentes et toponymie des quartiers à Tokombéré. Sociétés et jeunesses en difficulté [En ligne], $n^{\circ}$ 11. URL: http://journals.openedition.org/sejed/7203

Giraut F., Rochefort M., 2006. Discussion la marginalité socio-spatiale : une notion à déconstruire dans le contexte des villes du sud ? Revue Tiers Monde, $n^{\circ} 185$, p. 14-16.

Giraut F. et al., 2008. Au nom des territoires ! Enjeux géographiques de la toponymie. L'espace géographique, vol. 37, $\mathrm{n}^{\circ}$ 2, p. 97-105.

Giraut F. et al., 2008. Enjeux de mots : les changements toponymiques Sud-Africains. L'espace géographique, vol. 37, $\mathrm{n}^{\circ}$ 2, p. 131-150.

Giraut F., Antheaume B, 2012. Toponymy of power, power of toponymy? Colonial and contemporary Togolese place renaming. In 5th Trends in Toponymy Conference. Université de Bern. 2012. URL: https://archive-ouverte.unige.ch/unige:22883

Giraut F., Houssay-Holzschuch M., 2019. Au-delà du toponyme, la dimension politique de la territorialisation par la nomination. In Pasquali S., Gonzalez J., Au-delà du toponyme. Approches interdisciplinaires de la territorialité. Egypte et Méditerranée anciennes. Montpellier, ENiM.

Goerg, O., 2006. Domination coloniale, construction de «la ville » en Afrique et dénomination. Afrique histoire, vol. 5, $\mathrm{n}^{\circ}$ 1, p. 15-45.

Guillorel H., 2013. Onomastique, marqueurs identitaires et plurilinguisme. Les enjeux politiques de la toponymie et de l'anthroponymie. Droit et cultures [En ligne], n 64. URL: http:// journals.openedition.org/droitcultures/2780

Guyot S., Seethal C., 2007. Identity of place, places of identities: change of place names in postapartheid South Africa. South African Geographical Journal, vol. 89, nº 1, p. 55-63.

Houssay-Holzschuch M., 2008. Nomen est nomen : lecture des changements toponymiques. L'espace géographique, vol. 37, $\mathrm{n}^{\circ}$ 2, p. 153-159.

Hugues M M., 2020. Agriculture urbaine et trajectoires d'accès au foncier pour les citadins précarisés au Cameroun : l'agriurbain comme perspective stratégique. Territoire en mouvement [En ligne], nº 44-45. URL: http://journals.openedition.org/tem/6411 - DOI: https://doi.org/10.4000/ tem.6411

Jodelet D., 1989. Les Représentations sociales. Paris, PUF, 424 p.

Joseph R., 1986. Le mouvement nationaliste au Cameroun. Les origines sociales de l'UPC. Paris, Karthala, $414 \mathrm{p}$. 
Leimdorfer, F., et al., 2002. Nommer les quartiers d'Abidjan. In Topalov C. (éd), Les divisions de la ville. Paris, Éditions de la Maison des sciences de l'homme, p. 313-346.

Leques R., 1955. La rébellion dans le pays bamiléké. Europe-France Outre-Mer, nº 305.

Mbembe A., 1989. L'État-historien. In Um Nyobe R., Ecrits sous maquis. Paris, L'Harmattan, p. 7-42.

Mbembe A., 1996. La naissance du maquis dans le Sud-Cameroun (1920-1960) : histoire des usages de la raison en colonie. Paris, Karthala, 438 p.

Ndjock Nyobe I. P., 2014. L'héritage colonial à l'épreuve de la mémoire : le cas du monument Leclerc à Douala, Yaoundé. In Tchumtchoua E., Dikoume A.-F. (dir.), Douala : histoire et patrimoine. Yaoundé, Editions Clé.

Ndock Ndock G., 2020. Cultiver d'abord et habiter après : l'agriculture périurbaine comme stratégie d'appropriation foncière dans l'arrière-pays de Yaoundé. Territoire en mouvement. Revue de géographie et aménagement [En ligne], nº 44-45. URL: http://journals.openedition.org/tem/6257 - DOI: https://doi.org/10.4000/tem.6257

Ndock Ndock G., Assako Assako R.J., Yapi-Diahou A., 2015. Métropolisation de Yaoundé et mutations des structures foncières à Soa, ville satellite de Yaoundé. Le Journal des Sciences sociales, numéro spécial mars, p. 77- 83.

Njoh A. J., 2010. Toponymic inscription, physical addressing and the challenge of urban management in an era of globalization in Cameroon. Habitat International, vol 34, $\mathrm{n}^{\circ} 4$, p. 427-435. Njoh, A. J., 2017. Toponymic Inscription as an Instrument of Power in Africa: The case of colonial and post-colonial Dakar and Nairobi. Journal of Asian and African Studies, vol. 52, $\mathrm{n}^{\circ}$ 8, p. 1174-1192.

Pangop Kameni A. C., 2006. Politiques de toponymie urbaine au Cameroun : adresses ou maladresses dans les villes? [disponible sur www.cidegef.refer.org/douala/pangop_alain.doc consulté le 16 avril 2017).

Paquot T., 2010. Les faiseurs de villes. Infolio.

Stadnicki R., 2009. Nouvelles centralités et recompositions socio-spatiales dans le grand Sanaa (Yémen), Thèse de doctorat. Université François Rabelais, Tours, $581 \mathrm{p}$.

Tchumtchoua E., Ndjock Nyobe I. P., 2013. Le retour du refoulé colonial à l'échelle nationale : enjeux historiques et mémoriels des cinquantenaires. Actes du Colloque du Cinquantenaire de la Réunification du Cameroun. Douala, PDU.

Trosa S., 1995. Moderniser l'administration, comment font les autres? Approche comparative des stratégies de modernisation des administrations en France, au Royaume Uni et en Australie. Les éditions d'Organisation, $316 \mathrm{p}$.

Veschambre V., 2004. Appropriation et marquage symbolique de l'espace : quelques éléments de réflexion. Eso, $\mathrm{n}^{\circ}$ 21, p. 73-77.

Wanjiru, M. W., Matsubara K., 2016. Slum toponymy in Nairobi: A cultural arena for sociopolitical justice and symbolic resistance. International Planning History Society Proceedings, vol. 17 $\mathrm{n}^{\circ} 4$.

Wanjiru, M. W., Matsubara K., 2017. Slum toponymy in Nairobi, Kenya. A case study analysis of Kibera, Mathare and Mukuru. Urban and Regional Planning Review, ${ }^{\circ}$ 4, p. 21-44. 


\section{NOTES}

1. La restructuration urbaine est comprise ici au sens de la loi $n^{\circ} 2004 / 003$ du 21 avril 2004 régissant l'urbanisme au Cameroun. Elle est définie comme «un ensemble d'actions d'aménagements sur des espaces bâtis de manière anarchique, dégradés ou réalisés en secteur ancien, destinés à l'intégration d'équipements déterminés ou à l'amélioration du tissu urbain des agglomérations » (article 53, al.1).

2. Essi Gérard, Chef cellule de développement urbain de la CUY. Entretien réalisé par Ferdinand Mben Lissouck, 20 octobre 2009.

3. Le contrat de désendettement et de développement (C2D) est un outil qui permet de reconvertir la dette de certains pays en programmes de lutte contre la pauvreté.

4. Selon la loi portant code de l'urbanisme Le Certificat d'Urbanisme, L'autorisation de Lotir, Le Permis d'Implanter, Le Permis de Construire, Le Permis de Démolir, Le Certificat de Conformité, on pourrait ajouter l'absence ou la non réactualisation d'un Plan Directeur d'Urbanisme (PDU), Le Plan d'Occupation des Sols (POS), Le Plan de Secteur (PS), Les Plans Sommaires d'Urbanisme (PSU), etc.

5. Essi Gérard, Chef cellule de développement urbain de la CUY. Entretien réalisé par Ferdinand Mben Lissouck, 20 octobre 2009.

\section{RÉSUMÉS}

Ce travail questionne les sources et enjeux du conflit de référents dans la dénomination des voies et des quartiers de Yaoundé. La polyphonie constatée est liée à la coexistence des registres populaire et officiel de production urbaine. Le premier supplée et précède l'urbanisme officiel en zones de marge, interstitielle ou non aedificandi. Les référents toponymiques $\mathrm{du}$ registre populaire sont associés à des repères pratiques d'orientation. Ils expriment la mémoire ou la permanence des lieux appropriés et symboles d'urbanité pour les citadins des milieux populaires. Cette toponymie urbaine de pratique vernaculaire peut se heurter aux projections toponymiques fonctionnelles et mémorielles du registre officiel. En situation d'urbanisme de rattrapage, les lieux et quartiers sont rebaptisés selon divers référents et symboles qui rappellent pouvoir, histoire et culture officielles. Ce dépaysement et l'assujettissement induits des citadins conduisent au rejet de l'odonymie officielle, d'où le dualisme observé.

This paper examines the sources and stakes of the conflict of referents in the naming of roads and neighbourhoods in Yaoundé. This observed polyphony is attributed to the coexistence of popular and official registers of urban production. The first one overrides and precedes official urban planning in marginal, interstitial or non aedificandi zones. Toponymic referents of popular register are associated with practical reference points for orientation. They express the memory or perennity of appropriate places and symbols of urbanity for city-dwellers in populated areas. This urban toponymy of vernacular practice can clash with the functional and memory-oriented toponymic projections of official register. In a remedial urban planning situation, places and districts are renamed according to various referents and symbols which recall official power, history and culture. This disorientation and subjugation of city-dwellers brings about the rejection of official odonymy, resulting in the dualism which is observed. 
INDEX

Keywords : popular urbanism, remedial urbanism, naming, odonymy conflict

Mots-clés : urbanisme populaire, urbanisme de rattrapage, adressage, toponymie, conflit d'odonymie

Thèmes : Sur le Champ - Sur le Terrain

\section{AUTEUR}

\section{GASTON NDOCK NDOCK}

Gaston Ndock Ndock, nndock@yahoo.fr, est chargé de Cours, à l'École Normale Supérieure de Yaoundé, Université de Yaoundé 1 (Cameroun). Il a récemment publié :

- Ndock Ndock G, 2020. Cultiver d'abord et habiter après : l'agriculture périurbaine comme stratégie d'appropriation foncière dans l'arrière-pays de Yaoundé. Territoire en mouvement. Revue de géographie et aménagement [En ligne]. URL: http://journals.openedition.org/tem/6257 - DOI: https://doi.org/10.4000/tem.6257

- Ndock Ndock G, Assako Assako R.J., Yapi-Diahou A, 2015. Métropolisation de Yaoundé et mutations des structures foncières à Soa, ville satellite de Yaoundé. Le Journal des Sciences sociales, numéro spécial-mars 2015, p. 77- 83.

- Mbaha J.P., Ndock Ndock G., 2015. Le Nkam face à la métropolisation doualaise, entre ponction, réfraction au développement et faiblesse de la participation. In Elong J. G., Tchawa P., Moupou M. (ed), Participation et développement rural au Cameroun. Éditions Clé, p. 238-247. 